\title{
ANÁLISE DESCRITIVA DOS PERFIS SOCIAL, CLÍNICO, LABORATORIAL E ANTROPOMÉTRICO DE PACIENTES COM DOENÇAS INFLAMATÓRIAS INTESTINAIS, INTERNADOS NO HOSPITAL UNIVERSITÁRIO CLEMENTINO FRAGA FILHO, RIO DE JANEIRO
}

\author{
Paula Peruzzi ELIA ${ }^{1}$, Homero Soares FOGAÇA², Rodrigo G. G. Rego BARROS ${ }^{3}$, Cyrla ZALTMAN² \\ e Celeste Siqueira C. ELIA ${ }^{2}$
}

RESUMO - Racional - Estudos epidemiológicos sobre doenças inflamatórias intestinais no Brasil são limitados devido a dificuldades diagnósticas e reduzidas amostras populacionais estudadas. A maioria dos estudos de sua prevalência disponível é composta por amostras de pacientes sob acompanhamento em ambulatório, entretanto a análise do perfil de pacientes com doenças inflamatórias intestinais hospitalizados pode auxiliar na detecção de marcadores preditivos de sua gravidade, o que permitirá intervenções médicas precoces visando a redução da taxa de hospitalização e os gastos do sistema de saúde. Objetivos - Descrever o perfil social, clínico, laboratorial e antropométrico dos pacientes adultos com doenças inflamatórias intestinais internados em hospital universitário terciário. Métodos - Estudo prospectivo com 43 pacientes com doenças inflamatórias intestinais internados nas enfermarias clínicas e cirúrgicas e no setor de emergência do Hospital Universitário Clementino Fraga Filho da Universidade Federal do Rio de Janeiro, RJ. Foram caracterizados dados demográficos, presença de co-morbidades, localização e comportamento clínico, história cirúrgica, manifestações extra-intestinais utilizando-se definições padronizadas. Os resultados laboratoriais foram verificados nos prontuários e as medidas antropométricas foram realizadas durante a entrevista. Resultados - A maioria dos pacientes apresentou doença de Crohn ( $72,1 \%$ ), com localização íleo-colônica (60\%) e com comportamento penetrante (77,4\%), enquanto no grupo retocolite ulcerativa idiopática predominou a pancolite (50\%). No grupo total de retocolite ulcerativa idiopática, a artralgia foi a manifestação extra-intestinal mais freqüente (44,2\%) e 97,7\% já havia apresentado algum tipo de complicação relacionada à doença de base. Apesar do uso de terapêutica específica para doenças inflamatórias intestinais prévio à hospitalização em 79,1\% dos enfermos, as causas mais freqüentes de internação estavam relacionadas à atividade de doença. A presença de co-morbidades só ocorreu em $30,2 \%$. Conclusão - Os pacientes com doenças inflamatórias intestinais internados nas enfermarias do Hospital apresentam doença grave, em atividade, com complicações e manifestações extra-intestinais freqüentes, apesar do uso prolongado de corticóide. A doença de Crohn, por apresentar evolução mais agressiva, representou a maior parcela dos pacientes internados. A hipoalbuminemia, a anemia e as alterações antropométricas são comuns nos pacientes hospitalizados, podendo estar relacionadas a sua maior gravidade evolutiva.

DESCRITORES - Doença de Crohn. Proctocolite. Hospitalização.

\section{INTRODUÇÃO E LITERATURA}

A retocolite ulcerativa idiopática (RCUI) e a doença de Crohn (DC), também chamadas de doenças inflamatórias intestinais (DII), compõem um grupo heterogêneo de doenças cuja manifestação final comum é a inflamação, em cuja patogênese estão envolvidos diferentes fatores genéticos, imunológicos e ambientais ${ }^{(10)}$.

As manifestações clínicas são decorrentes tanto do acometimento do trato gastrointestinal (TGI) pela doença, como pelo surgimento de manifestações extra-intestinais, que ocorrem em cerca de $35 \%$ dos pacientes ${ }^{(13,33)}$.

Trabalho realizado no Serviço de Gastroenterologia do Hospital Universitário Clementino Fraga Filho da Universidade Federal do Rio de Janeiro, RJ.

${ }^{1}$ Serviço de Endoscopia Digestiva Pediátrica - Departamento de Cirurgia Pediátrica do Instituto Fernandes Figueira - FIOCRUZ; ${ }^{2}$ Disciplina de Gastroenterologia, da Universidade Federal do Rio de Janeiro; ${ }^{3}$ Faculdade de Medicina da Universidade Federal do Rio de Janeiro, RJ.

Correspondência: Dra. Paula Peruzzi Elia - Rua Visconde de Pirajá, 228 - apt.404 - Ipanema - 22410-000 - Rio de Janeiro, RJ. E-mail: paulaelia@terra.com.br paulaelia@iff.fiocruz.br 
Embora de etiologia multifatorial, muitos pacientes internados com exacerbação aguda de sua doença apresentam desnutrição protéico-calórica $^{(6,11,17,18)}$, hipoalbuminemia ${ }^{(4,7)}$ e anemia ${ }^{(13,16,33)}$. Leucocitose, trombocitose, aumento nos níveis séricos de proteína C reativa e de velocidade de hemossedimentação (VHS) podem ser decorrentes de inflamação crônica ${ }^{(3,33)}$.

O aumento da incidência das DII tem sido associado com maior grau de industrialização das regiões estudadas e à ocidentalização no estilo de vida, incluindo hábitos alimentares e tabagismo ${ }^{(24,29)}$.

Acometem pacientes jovens e economicamente ativos e apresentam alta morbidade. Isso representa grande custo econômico para os indivíduos e para a saúde pública devido ao uso prolongado de medicamentos, necessidade de inúmeros e complexos exames diagnósticos, freqüentes internações hospitalares e, muitas vezes, realização de cirurgias.

No Brasil são poucos os estudos epidemiológicos que permitem conhecer a incidência e a prevalência das DII. No entanto, essas enfermidades não são tão raras como se acreditava há alguns $\operatorname{anos}^{(9)}$. A maior parte dos estudos é realizada em pacientes de ambulatório, sendo de extrema importância conhecer os aspectos epidemiológicos, clínicos e nutricionais dos pacientes internados, a fim de que se possa realizar o diagnóstico precoce e iniciar terapêutica mais adequada, retardando ou impedindo o desenvolvimento de suas possíveis complicações.

\section{MÉTODOS}

Foram estudados 43 pacientes com diagnóstico de DII internados nas enfermarias de gastroenterologia, coloproctologia, cirurgia geral, clínica médica e emergência do Hospital Universitário Clementino Fraga Filho da Universidade Federal do Rio de Janeiro (HUCFF-UFRJ), no período compreendido de março de 2001 a junho de 2004. Nesse período, 382 pacientes com DII estavam sendo acompanhados ativamente nos ambulatórios de gastroenterologia e coloproctologia do hospital.

Critérios de inclusão:

a) diagnóstico de DII comprovado através de critérios clínicos, radiológicos, endoscópicos e/ou histopatológicos;

b) o motivo de internação estar relacionado com a atividade das DII, suas complicações, ou ambas.

Critérios de exclusão:

a) idade abaixo de 16 anos ou acima de 75 anos;

b) presença de colites por outras causas, incluindo infecções parasitárias;

c) presença de outras doenças granulomatosas, como a tuberculose;

d) diagnóstico de doenças neoplásicas;

e) portadores do vírus HIV ou pacientes com AIDS;

f) enfermos restritos ao leito;

g) gestantes ou nutrizes;

h) pacientes internados por complicações da terapêutica das DII;

i) enfermos com insuficiência orgânica múltipla.

Todos os pacientes, após esclarecimento verbal e preenchimento de consentimento informado, foram submetidos ao protocolo de investigação. O trabalho foi aprovado pela comissão de Ética Médica do HUCFF-UFRJ.

Foram excluídos sete pacientes ao longo deste estudo. As causas de exclusão foram as seguintes: um paciente acamado com necrose asséptica da cabeça do fêmur, dois com diagnóstico de tuberculose intestinal, uma com diagnóstico de endometrioma e outra com tumor carcinóide multicêntrico, um enfermo tinha tumor de reto e o outro estava internado para se investigar um nódulo pulmonar.

\section{Anamnese}

Os dados analisados foram: identificação, diagnóstico principal e secundário, data de início de sintomas e de diagnóstico da doença, diferença de tempo entre o início dos sintomas e do diagnóstico, complicações relacionadas às DII, manifestações extra-intestinais, tratamento medicamentoso e/ou cirúrgico em ambulatório e internado, motivo da internação e duração desta, e a procedência do paciente. Avaliou-se, também, o hábito tabágico, uso de antiinflamatórios não-esteróides (AINE), história familiar de alguma doença intestinal, a necessidade de hemotransfusão e de suporte nutricional durante a hospitalização.

Os aspectos sociais estudados foram: residência, naturalidade, nível de escolaridade, saneamento básico do local de moradia, renda mensal individual, familiar e per capita.

A extensão das DII e o comportamento da DC foram descritos conforme a classificação de Viena ${ }^{(16)}$. Os índices de atividade de doença utilizados foram HARVEY e BRADSHAW ${ }^{(19)}$ para DC e LICHTIGER et al. ${ }^{(23)}$ para RCUI.

\section{Avaliação antropométrica}

Foi realizada pelos mesmos profissionais, com experiência prévia nesse tipo de mensuração. Foram avaliados: peso corporal $(\mathrm{kg})$, estatura $(\mathrm{m})$, prega cutânea tricipital (PCT), índice de massa corporal de Quelet (IMC), índice de gordura do braço (IGB), circunferência muscular do braço e percentagem de perda de peso (\%PP).

Utilizaram-se as seguintes fórmulas para cálculo ${ }^{(12)}$ :

$$
\begin{aligned}
& \mathrm{IMC}=\frac{\text { peso atual }(\mathrm{kg})}{\text { estatura }\left(\mathrm{m}^{2}\right)} \\
& \mathrm{CMB}(\mathrm{cm})=\mathrm{CB}(\mathrm{cm})-(0,0314 \times \mathrm{PCT}(\mathrm{mm}) \\
& \mathrm{PP}=\frac{\text { peso usual }(\mathrm{PU})-\text { peso atual }(\mathrm{PA})}{\text { peso usual }(\mathrm{PU})} \times 100
\end{aligned}
$$

Considerou-se como peso usual do paciente aquele referido 6 meses antes da internação os e como perda de peso significativa, valores maiores que $10 \%$ em período de 6 meses.

\section{Avaliação laboratorial}

Os exames laboratoriais foram coletados com prazo máximo de 7 dias antes ou após a realização da avaliação antropométrica. Foram realizadas a dosagem sérica de proteínas totais e albumina, ferritina, hemoglobina e proteína $\mathrm{C}$ reativa, a contagem de linfócitos totais e a medida da VHS. 


\section{Metodologia estatística}

Para comparação dos dados quantitativos entre dois grupos foi utilizado o teste de Mann-Whitney e para comparação de dados qualitativos o teste exato de Fisher. Foram aplicados testes não-paramétricos para as variáveis que não apresentassem distribuição normal. O critério de determinação de significância adotado foi o nível de 5\%. A análise estatística foi processada pelo software estatístico $\mathrm{SAS}^{\circledR}$ System.

\section{RESULTADOS}

Trinta e um enfermos $(72,1 \%)$ tinham o diagnóstico de DC, $10(23,3 \%)$ de RCUI e $2(4,7 \%)$ de colite indeterminada. As idades variaram entre 16 e 74 anos, com média de 36,7 e mediana de 34 anos. Trinta pacientes eram mulheres $(69,8 \%)$ e 13 homens $(30,2 \%)$. Trinta e um eram brancos $(72,1 \%)$, sendo 9 pardos $(20,9 \%)$ e 3 (7\%) negros.

Quanto à extensão da doença, nos pacientes com DC: $60 \%$ era ileocólica, $20 \%$ cólica e $20 \%$ restrita ao íleo terminal. Em relação à RCUI: $50 \%$ apresentavam pancolite, $30 \%$ sigmóideproctite e $20 \%$ colite esquerda. Ao se avaliar o comportamento da DC, predominou a doença penetrante em 24 casos $(77,4 \%)$, seguida de doença estenosante em $4(12,9 \%)$ e em $3(9,7 \%)$ doença inflamatória (não-estenosante e não-penetrante).

O período entre o início dos sintomas das DII e o diagnóstico foi menor que 1 ano em 72,5\% dos pacientes. Apenas um enfermo com DC nunca havia tido complicação e 51,6\% daqueles com DC já tinham apresentado mais de três complicações (Figura 1).

As manifestações extra-intestinais que os pacientes com DII já haviam apresentado em algum momento da evolução de sua doença estão descritas na Figura 2.

Constatou-se que $69,8 \%$ dos pacientes não tinham outras doenças associadas às DII. A enfermidade mais freqüentemente associada com a DC foi a hipertensão arterial sistêmica (12,9\%), enquanto na RCUI observou-se associação com hepatopatias (dois casos de colangite esclerosante primária, um de hepatopatia crônica em investigação e um de esquistossomose).

Alguns pacientes avaliados já haviam sido submetidos a cirurgias devido às DII, especialmente aqueles com DC (44,2\%), enquanto na RCUI apenas um (10\%) havia sido operado antes da internação (hemorroidectomia).

Em relação às cirurgias realizadas durante a hospitalização, a mais freqüente foi a ressecção intestinal na DC (nove pacientes), seguida da fistulectomia (dois). Na RCUI, a cirurgia mais freqüente foi a colectomia total (dois).

O corticóide foi o medicamento mais utilizado antes $(67,7 \%$ dos pacientes com DC e $60 \%$ daqueles com RCUI) e durante a internação (80,6\% dos enfermos com DC e 90\% daqueles com RCUI), sendo que $51,6 \%$ dos pacientes com DC e $30 \%$ daqueles com RCUI faziam uso de corticóide por um período maior do que 6 meses. Considerando-se ainda as medicações em uso antes da internação, o grupo de imunomoduladores foi o segundo mais utilizado na DC $(25,8 \%)$ enquanto que na RCUI a sulfassalazina foi utilizada na mesma freqüência dos corticoesteróides $(60 \%)$.

$\mathrm{O}$ motivo da internação nos pacientes com DII avaliados está descrito na Figura 3.

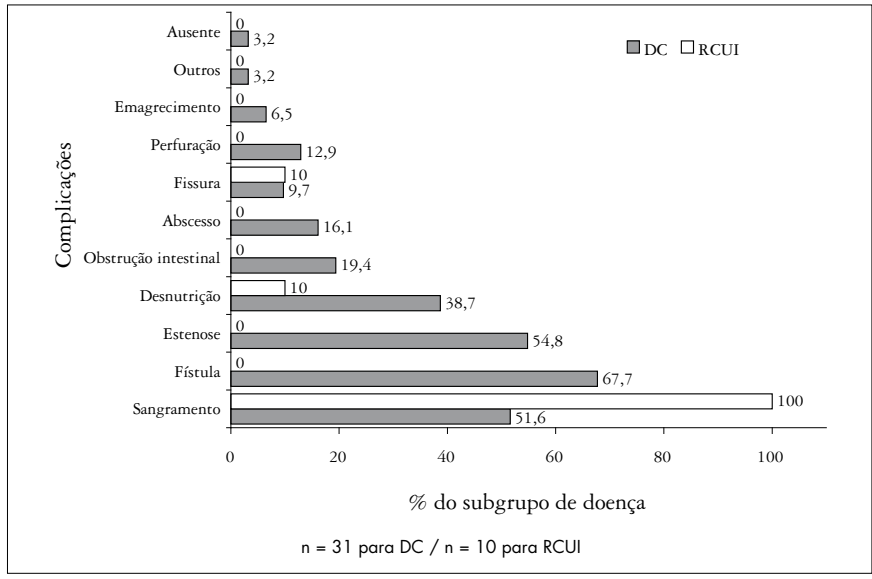

FIGURA 1. Complicações apresentadas pelos pacientes internados nas enfermarias de gastroenterologia do HUCFF-UFRJ, considerando subgrupos DC e RCUI

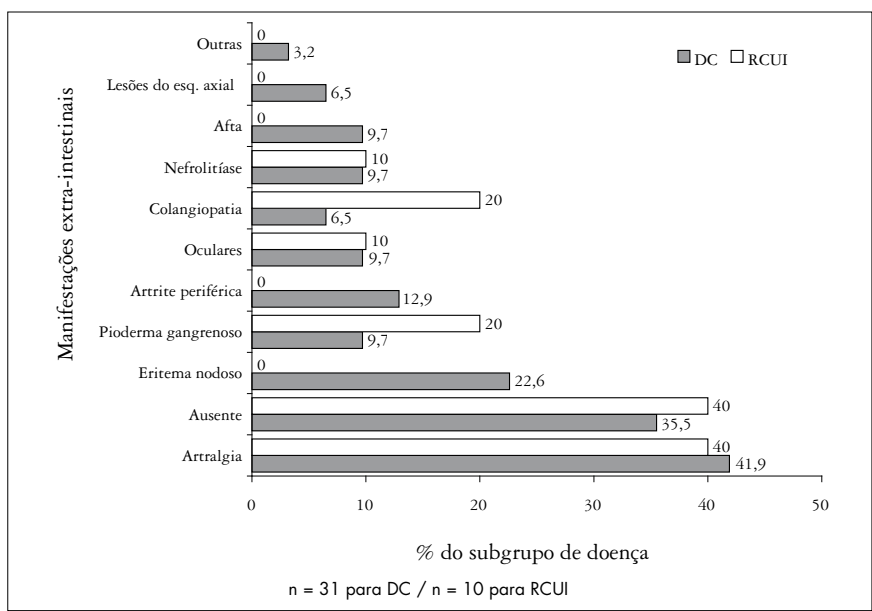

FIGURA 2. Manifestações extra-intestinais apresentadas pelos pacientes com DII internados nas enfermarias do HUCFF-UFRJ, considerando subgrupo DC e RCUI

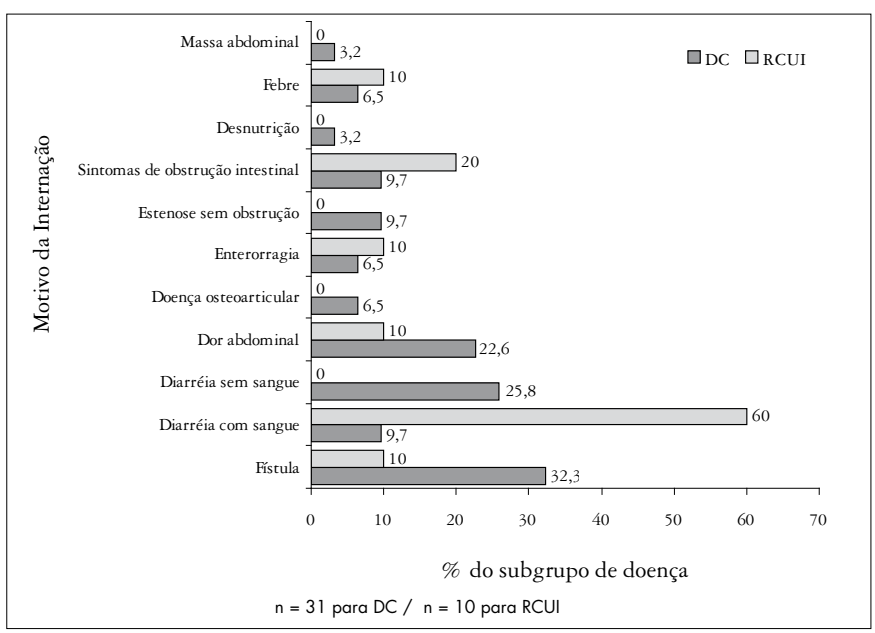

FIGURA 3. Motivo da internação dos pacientes com DII avaliados nas enfermarias do HUCFF-UFRJ, considerando os subgrupos DC e RCUI 
O tempo de internação mínimo foi de 8 dias e máximo de 94 , com média de 26,09 dias. Vinte e nove pacientes foram provenientes dos ambulatórios do HUCFF-UFRJ $(67,4 \%)$, 3 da emergência deste hospital (7\%) e $11(25,6 \%)$ vieram de outra instituição.

Sete pacientes eram ex-tabagistas (16,3\%) e sete $(16,3 \%)$ ainda fumavam. Apenas nove (20,9\%) estavam em uso de AINE antes da internação e $93 \%$ desconheciam a história familiar de doenças intestinais. Não foi possível estabelecer associação estatística entre o fumo e o diagnóstico de $\mathrm{DC}$ ou de RCUI $(P=0,99)$, assim como correlacionar o uso de AINE e a atividade de doença, e a história familiar de enfermidades intestinais com o diagnóstico das DII.

Durante a hospitalização, $20,9 \%$ dos pacientes não necessitaram de hemotransfusão e dois $(4,7 \%)$ faleceram.

Em relação aos índices de atividade de doença, observouse que havia doença ativa em $50 \%$ dos enfermos com RCUI, conforme o índice de Lichtiger, e 80,7\% dos com DC, de acordo com o índice de Harvey e Bradshaw.

Dentre as alterações laboratoriais, a que predominou foi a hipoalbuminemia tanto na DC quanto na RCUI, conforme descrito na Figura 4. As alterações antropométricas, foram freqüentes e multifatoriais (Figura 5) e ocorreram tanto na DC quanto na RCUI, no entanto, não foi possível encontrar associação com significância estatística entre essas e o diagnóstico das DII, e entre essas e hipoalbuminemia.

Observou-se que $93 \%$ dos pacientes residiam no Grande Rio, sendo que $72,1 \%$ eram naturais do sudeste, $23,3 \%$ do nordeste, $2,3 \%$ do norte e $2,3 \%$ do sul.

Em relação à escolaridade, a maioria dos pacientes havia cursado apenas o ensino fundamental $(37,3 \%)$ e apenas um enfermo $(2,3 \%)$ havia completado o ensino superior.

Todos os doentes relataram saneamento básico em sua residência.

Em relação à renda mensal individual, observou-se que era ausente em 13 pacientes $(30,2 \%), 7(16,3 \%)$ recebiam até 1 salário mínimo, $16(37,2 \%)$ entre 1 e 5 salários, 4 (9,3\%)

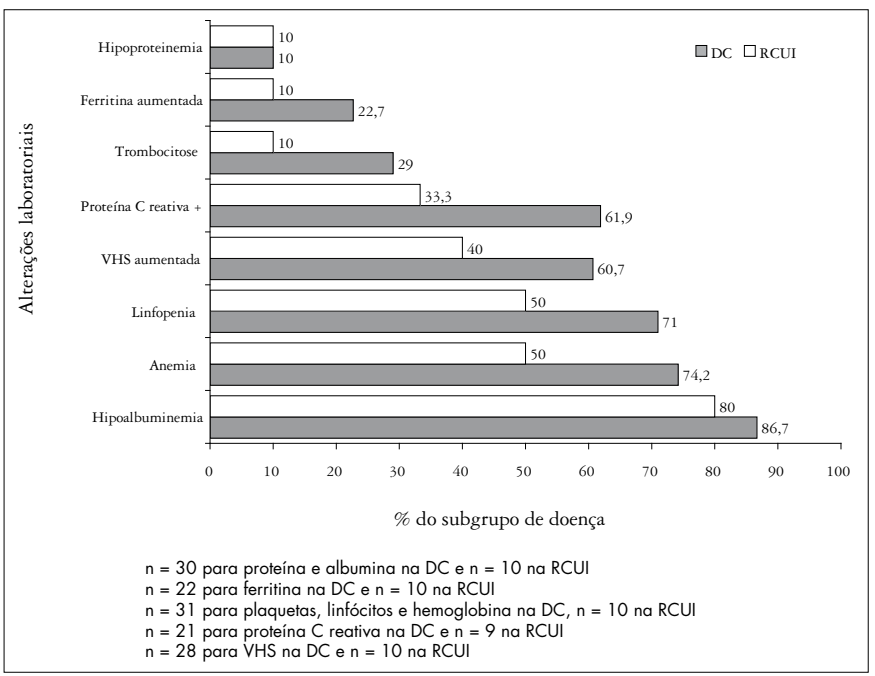

FIGURA 4. Alterações laboratoriais dos pacientes internados com DII nas enfermarias do HUCFF-UFRJ, considerando os subgrupos DC e RCUI entre 6 e 10 salários e não foi possível determinar a renda em 3 doentes $(7 \%)$. Em relação à renda mensal familiar, viu-se que 2 famílias (4,7\%) ganhavam até 1 salário mínimo, 23 (53,5\%) entre 1 e 5 salários, 6 (14\%) entre 6 e 10 salários, $6(14 \%)$ mais de 10 salários e em 6 casos (14\%) não foi possível determinar a renda mensal familiar.

Considerando a renda mensal familiar per capita, observou-se que não havia nenhum caso de ausência de rendimentos, $41,9 \%$ (18 famílias) recebiam até 1 salário, $41,9 \%$ entre 1 e 5, apenas 1 família $(2,3 \%)$ recebia entre 6 e 10 salários mínimos, e não houve casos com mais de 10 salários, em 6 pacientes (14\%) esta avaliação foi impossibilitada.

\section{DISCUSSÃO}

Embora a incidência da RCUI seja maior que a da $\mathrm{DC}^{(10)}$, $72,1 \%$ dos pacientes do presente estudo tinham DC, o que pode ser justificado por se estar avaliando doentes internados e a DC tende a ter evolução mais grave, com mais complicações intestinais, hospitalizações e intervenções cirúrgicas que a RCUI ${ }^{(26)}$. O período de internação foi considerado longo, com importante ônus para a rede pública de saúde.

A distribuição da $\mathrm{DC}$ foi muito semelhante à descrita na literatura ${ }^{(11)}$, com acometimento do íleo terminal mais freqüentemente. No entanto, contrariando dados da literatura ${ }^{(22)}$, observou-se que metade dos pacientes com RCUI apresentava pancolite, provavelmente por se estar estudando pacientes internados, com uma apresentação mais agressiva.

Embora as DII sejam doenças raras e necessitem de exames complexos para o seu diagnóstico, entre os pacientes internados o intervalo de tempo entre o início de sintomas e o diagnóstico foi menor que 1 ano em $72,5 \%$ dos casos, sendo que a maioria referia período inferior a 6 meses. Esse achado pode representar maior conscientização da existência das DII no Brasil, maior acessibilidade às técnicas diagnósticas ${ }^{(24)} \mathrm{e}$ ao fato de enfermos com evolução grave estarem sendo encaminhados

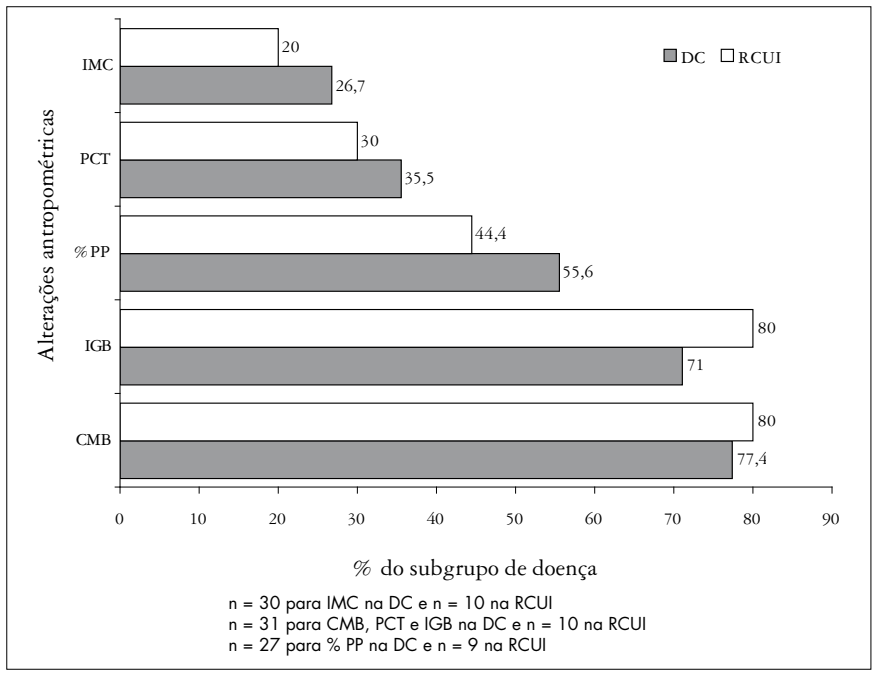

FIGURA 5. Alterações antropométricas dos pacientes com DII internados nas enfermarias do HUCFF-UFRJ, considerando os subgrupos DC e RCUI 
mais rapidamente para internação em um hospital de referência, como o HUCFF-UFRJ.

Apenas um doente avaliado ainda não tinha desenvolvido complicações relacionadas às DII e $37,2 \%$ (todos com DC) já tinham apresentado mais de três complicações em algum momento da evolução da doença. Observou-se mais que o dobro na freqüência de fistulização $(77,42 \%)$ se comparada à descrita por REGUEIRO $(30 \%)^{(27)}$.

Outro achado interessante é que a maioria destes pacientes desenvolveu o início dos sintomas antes dos 40 anos de idade. $\mathrm{O}$ início precoce dos sintomas, conforme a classificação de Viena ${ }^{(15)}$, tem relação com um curso mais agressivo, acometimento do íleo terminal, fístulização e estenoses mais freqüentes.

Em relação às manifestações extra-intestinais, atentou-se que, semelhante à casuística mundial ${ }^{(13,33)}$, a artralgia foi a mais freqüente, o eritema nodoso ocorreu apenas na DC $(22,6 \%)$ e o pioderma gangrenoso predominou na RCUI $(20 \%)$. A nefrolitíase foi a complicação urogenital mais comum e apenas $9,3 \%$ dos pacientes apresentaram manifestações oculares. A única manifestação hepatobiliar encontrada foi a colangiopatia, embora a esteatose hepática seja descrita como a manifestação hepatobiliar mais comum nas DII ${ }^{(33)}$, o que sugere que esta tenha sido pouco diagnosticada por ser uma manifestação assintomática, sem investigação diagnóstica rotineira.

A maioria dos pacientes não apresentava co-morbidades $(69,8 \%)$, provavelmente por se tratar de população jovem.

Apenas $20,9 \%$ dos enfermos avaliados não estavam em uso de medicamentos antes da hospitalização, destes, 77,7\% estavam sem tratamento devido à ausência de diagnóstico formal das DII até o momento da internação. A droga mais utilizada antes e durante a internação foi o corticóide, por mais de 6 meses em 51,6\% dos pacientes com DC e em 30\% daqueles com RCUI. Os imunomoduladores estavam sendo administrados em menores proporções, provavelmente por não serem considerados medicamentos de primeira escolha na fase aguda das $\mathrm{DII}^{(5,8,20,31,32)}$. Apesar da alta incidência de fístulas e de atividade de doença, apenas um paciente estava em uso de infliximabe, podendo estar diretamente relacionado com o nível socioeconômico dos indivíduos atendidos no HUCFFUFRJ, uma vez que se trata de medicamento de alto custo, que não era fornecido pelo Ministério da Saúde até o momento da realização do presente estudo.

Aspecto que chama a atenção é que, apesar da maioria dos enfermos $(79,1 \%)$ estarem em tratamento das DII antes da hospitalização, principalmente com corticóide, as causas mais freqüentes de internação estavam diretamente relacionadas à atividade de doença ou a complicações desta. Ao se avaliar o índice de HARVEY e BRADSHAW ${ }^{(19)}$ para DC e o de LICHTIGER et al. ${ }^{(23)}$ para RCUI, constatou-se que $80,7 \%$ dos pacientes com DC e $50 \%$ daqueles com RCUI estavam em atividade de doença. Estes dados corroboram a hipótese de que os doentes hospitalizados com DII apresentem doença grave, muitas vezes refratária ao tratamento clínico, associada a complicações de alta morbidade, representando elevado custo hospitalar.

As cirurgias, indicadas nos casos de falha no tratamento clínico ou na presença de sérias complicações da doença, podem ser curativas na RCUI, mas não na DC. A proctocolectomia é requerida em $15 \%$ dos enfermos, usualmente por doença refratária ${ }^{(22)}$. Na presente casuística, $20 \%$ do grupo com RCUI foram submetidos a colectomia total com ileostomia por refratariedade à terapêutica clínica. Na DC, a cirurgia está reservada ao tratamento das complicações e é mais freqüente quando há acometimento ileocecal $(87 \%$ a $90 \%)$ maior que colorretal $(50 \% \text { a } 58 \%)^{(3)}$. Em estudo realizado pelo Instituto de Gastroenterologia do Hospital das Clínicas, em Minas Gerais ${ }^{(9)}, 50 \%$ dos enfermos com DC haviam sido submetidos a, pelo menos, uma cirurgia abdominal. Observou-se que na presente amostra, as duas cirurgias mais freqüentemente realizadas nos enfermos com DC, antes e durante a hospitalização, foram ressecções intestinais e fistulectomia. A apendicectomia havia sido realizada em três doentes (7\%) antes da internação e deve ser ressaltado que, em alguns casos, a DC tem apresentação inicial que mimetiza a apendicite aguda, pelo acometimento inflamatório da região íleocecal ${ }^{(3)}$.

O nível de albumina sérica freqüentemente correlacionado ao estado nutricional, também pode refletir reação de fase aguda mediada por citocinas durante um evento inflamatório(4, 10). A desnutrição e a hipoalbuminemia são achados relevantes nas DII, especialmente na DC ${ }^{(2)}$. Encontrou-se hipoalbuminemia na maior parte dos pacientes com DC e RCUI. No entanto, uma minoria dos dois grupos apresentava concentração de proteína total sérica menor que $5 \mathrm{~g} / \mathrm{dL}$, reforçando a hipótese de não haver relação entre hipoalbuminemia e desnutrição na amostra estudada. Para confirmar este dado, tentou-se correlacionar individualmente cada alteração antropométrica com a hipoalbuminemia, mas não houve significância estatística entre nenhuma destas associações.

Anemia é outro achado relevante ${ }^{(7,8,13,16,36)}$ e foi observada em $69,8 \%$ dos doentes avaliados, sendo mais freqüente na DC, embora a enterorragia fosse mais comum na RCUI. Apesar da sua presença freqüente, uma minoria de pacientes foi hemotransfundida. Os resultados desta série sugerem que a anemia nas DII não é exclusivamente ferropriva por perda de sangue vivo ou oculto pelo TGI, corroborando a hipótese da sua etiologia ser multifatorial ${ }^{(16)}$. Soma-se a isto o fato de apenas um paciente ter apresentado dosagem sérica de ferritina baixa, que é um achado relevante na anemia ferropriva. Embora se saiba que a ferritina também seja uma proteína de fase aguda e na presença de inflamação, níveis séricos normais não excluem deficiência de ferro ${ }^{(20)}$. Logo, a dosagem sangüínea elevada de ferritina em $22,73 \%$ dos enfermos pode estar sendo subestimada.

A contagem sérica de linfócitos pode ser utilizada como um parâmetro nutricional para medir a competência imunológica ${ }^{(29)}$. No presente estudo, $71 \%$ dos pacientes com DC e $50 \%$ daqueles com RCUI apresentavam linfopenia, provavelmente por alteração do estado nutricional, além do efeito colateral dos imunossupressores, que estavam sendo utilizados em 30,2\% dos hospitalizados.

$\mathrm{O}$ aumento dos níveis séricos de VHS e da proteína $\mathrm{C}$ reativa, assim como a presença de trombocitose podem representar inflamação $0^{(3,21,33,34)}$. Nesta casuística, encontrou-se aumento na medida sérica de VHS $(60,71 \%$ dos doentes com DC e $40 \%$ com RCUI), de proteína C reativa (foi dosada em apenas 31 pacientes e estava positiva em $54,8 \%$ ) e trombocitose 
(25,81\% dos enfermos com DC e $10 \%$ com RCUI). No entanto, não foi possível fazer correlação estatística entre estes resultados e a atividade de doença.

Conforme o Inquérito Brasileiro de Avaliação Nutricional Hospitalar ${ }^{(35)}$, ficou demonstrado que $48 \%$ dos doentes internados estavam desnutridos. Nas DII, a desnutrição é achado comum, com múltiplas causas, acometendo $85 \%$ dos hospitalizados com exacerbação aguda destas enfermidades ${ }^{(18)}$. As medidas antropométricas são uma forma de mensuração de fácil aquisição, manuseio simples e baixo custo na avaliação do perfil nutricional ${ }^{(14)}$. Refletem o passado do estado nutricional e têm como limitação a impossibilidade de detectar alguns distúrbios nutricionais de instalação muito recente ou deficiências específicas de nutrientes ${ }^{(12)}$.

A percentagem de perda de peso recente $(\% \mathrm{PP})$ tem boa correlação com a morbidade e mortalidade ${ }^{(12)}$, pois inclui o tempo em que ocorreu a alteração ponderal. Na presente casuística, praticamente a metade dos enfermos avaliados já apresentava perda de peso importante pelo menos 6 meses antes da internação, o que pode ter contribuído para o agravamento da doença de base e piora clínica.

SANTOS et al. ${ }^{(30)}$ descreveram que o IMC é de grande utilidade para avaliar o estado nutricional quando em associação com outros indicadores, uma vez que leva em consideração o somatório de todos os compartimentos corporais, sem distinguir o peso associado ao músculo ou à gordura corporal. Neste grupo em estudo, as alterações do IMC ocorreram em $26,2 \%$ dos pacientes, percentagem menor que a das outras medidas antropométricas (IGB, CMB e \% PP). De modo que se fosse interpretada isoladamente, poderia subdiagnosticar os casos de desnutrição.

Em concordância com trabalho realizado por SANTOS et al. ${ }^{(30)}$, encontraram-se alterações na PCT e na CMB, mais freqüentes que as descritas no estudo citado, provavelmente porque se avaliaram pacientes internados e aquela pesquisa foi realizada em doentes de ambulatório.

Apesar da utilização prolongada de corticóide, que modifica o estado nutricional através do aumento do apetite e ganho ponderal ${ }^{(30)}$, havendo aumento da gordura corporal, houve redução do IGB em $72,1 \%$ dos enfermos ( $71 \%$ com DC e $80 \%$ com RCUI), ressaltando uma redução significativa na gordura corporal, independentemente da corticoterapia.

Embora tenha-se encontrado pelo menos uma alteração antropométrica na maioria dos pacientes desta série, apenas $25,8 \%$ dos com DC e $10 \%$ dos com RCUI usaram suplemento enteral, $6,45 \%$ com DC e 10\% com RCUI utilizaram suplemento nutricional oral. A nutrição parenteral total foi administrada em apenas dois doentes $(4,9 \%)$, sendo que ambos tinham sido submetidos a cirurgia de ressecção intestinal durante a internação. Pode-se justificar esta baixa percentagem de suporte nutricional pelo fato da avaliação do estado nutricional não ser ainda rotina nas enfermarias, sendo indicado em proporções menores do que o realmente necessário. Com o presente estudo, propõe-se que a avaliação antropométrica seja realizada rotineiramente nos enfermos com DII, uma vez que as diferentes mensurações antropométricas são de fácil manuseio, baixo custo e fidedignas quando realizadas por profissionais experientes, podendo contribuir para indicação mais precisa de suplementação nutricional em mais casos, reduzindo complicações infecciosas e pós-operatórias.

Ao se avaliar a distribuição quanto à idade, constatou-se que os doentes desta série são adultos jovens, com idade média de 36,7 e mediana de 34 anos. No entanto, esta distribuição não foi bimodal, como descrita em muitos centros ${ }^{(10)}$. Em relação à raça, $27,9 \%$ dos pacientes não eram da raça branca, o que pode ser justificado pela urbanização e assimilação de hábitos ocidentais, que levou a aumento no envolvimento de populações não-brancas ${ }^{(9,24,28)}$. Deve-se salientar que no Brasil há grande miscigenação racial e que, muitas vezes, encontram-se dificuldades em classificar a raça.

Existem alguns fatores considerados modificadores das DII, como o tabagismo (fator protetor para RCUI e fator de risco para DC) e o uso de AINE, que leva à exacerbação das DII ${ }^{(25)}$. Nesta casuística houve uma pequena amostra de pacientes tabagistas e em uso de AINE, não sendo possível correlacionar estes achados com a atividade de doença. Em relação à história familiar de enfermidades intestinais (história de DII, diarréia crônica sem diagnóstico e câncer intestinal), esta foi encontrada em apenas $7 \%$ dos pacientes.

Recentemente vem sendo descrita uma mudança no perfil epidemiológico das DII, notadamente devido à urbanização e à industrialização ${ }^{(24,28)}$. A maioria dos pacientes estudados (93\%) era proveniente do Grande Rio, que é um pólo industrial, e todos residiam em moradias com saneamento básico, sendo que $74,4 \%$ das residências tinham mais de três cômodos. Soma-se a isto o fato de que nenhum paciente era analfabeto e que $76,8 \%$ completaram o ensino fundamental. Apenas um completou o ensino superior.

Na avaliação da renda mensal individual, familiar e per capita, observou-se que 13 pacientes não tinham renda individual, mas nenhum declarou a ausência de renda familiar. Estes enfermos não tinham renda própria, provavelmente por estarem afastados de suas atividades devido à gravidade da doença. Isto demonstra o grande impacto das DII na renda familiar, limitando o aporte financeiro destes enfermos, sobrecarregando a família. Soma-se à morbidade desta enfermidade o fato de muitos pacientes desconhecerem os benefícios sociais e os direitos dos acometidos de doença crônica, de modo que, além de estarem afastados de suas atividades diárias, necessitam da compra de medicamentos caros.

Quanto à renda mensal per capita (o salário de todos os membros da família dividido pelo número de habitantes) verificou-se que 41,9\% (18 famílias) recebiam até 1 salário mínimo, 41,9\% recebiam entre 1 e 5 salários mínimos, e apenas 2,3\% (1 família) recebia entre 6 e 10 salários mínimos; em 14\% (6 famílias) esta renda foi indeterminada. Este dado se correlaciona perfeitamente com o perfil social da população brasileira e dos pacientes atendidos em hospitais públicos universitários, correspondendo a indivíduos de baixa renda, que dependem exclusivamente de atendimento fora do setor privado.

Apesar da gravidade da doença nos pacientes estudados, apenas dois (um com DC e um com RCUI) evoluíram para o óbito. Embora seja casuística muito pequena para ter significância estatística, pode-se observar que estes dois enfermos tinham em comum anemia, hipoalbuminemia acentuada e alterações antropométricas (\% PP, CMB e PCT). Estes três parâmetros 
isoladamente vêm sendo relacionados em alguns trabalhos com pior evolução da doença ${ }^{(4,16,25,30)}$. A hipoalbuminemia e a anemia seriam marcadores de gravidade, pois têm relação não apenas com atividade de doença, mas também com desnutrição. A desnutrição é conseqüência da própria enfermidade e contribui para a maior susceptibilidade do paciente a infecções e complicações pós-operatórias.

Estes exames laboratoriais de fácil realização e baixo custo estão disponíveis na maioria dos hospitais públicos. Os parâmetros antropométricos podem ser aferidos rotineiramente no ambulatório, por profissional treinado, sem prolongamento importante no tempo de consulta. A intervenção precoce a fim de se evitar ou tratar a desnutrição, permitiria uma abordagem adequada desta complicação e reduziria a morbidade, minimizando o número de internações e o custo com a terapêutica.

Seria interessante sugerir que estes três parâmetros, hipoalbuminemia, anemia e alterações antropométricas, fossem pesquisados rotineiramente no ambulatório e que o somatório desses achados pudesse ser interpretado como um marcador negativo na evolução clínica das DII.

\section{CONCLUSÃO}

Os pacientes com DII internados nas enfermarias do HUCFF-UFRJ apresentam quadro evolutivo mais grave, com a doença em atividade, na presença de freqüentes manifestações extra-intestinais, com ou sem complicações, apesar do uso prolongado de corticoterapia. A DC foi mais prevalente nos pacientes internados $(72,1 \%)$, por ser de evolução clínica mais agressiva se comparada à RCUI. A hipoalbuminemia, anemia $\mathrm{e}$ as alterações antropométricas são comuns nesta amostra e estão relacionadas a uma piora clínica das DII, podendo ser utilizados como parâmetro de avaliação de gravidade destas enfermidades. Entretanto a taxa de mortalidade é baixa. O perfil social não diferiu do encontrado na população estudada.

Elia PP, Fogaça HS, Barros RGGR, Zaltman C, Elia CSC. Descriptive analysis of the social, clinical, laboratorial and anthropometric profiles of inflammatory bowel disease inwards patients from the "Clementino Fraga Filho" University Hospital, Rio de Janeiro, RJ, Brazil. Arq Gastroenterol. 2007;44(4):332-9.

ABSTRACT - Background - The epidemiologic survey in Brazil is limited probably due to a diagnosis deficiency and a small number of population-based studies performed. The majority of the prevalence studies available have evaluated inflammatory bowel diseases outpatients, but the knowledge of the profile of inflammatory bowel diseases inpatients is important in order to detect predictive markers of disease severity that will allow earlier medical intervention decreasing the rate of hospitalization and reducing the Health System costs. Aim - To determine social, clinical, laboratorial and anthropometric profiles of hospitalized adults inflammatory bowel diseases patients of a tertiary university hospital. Methods - Prospective study was performed with 43 inflammatory bowel diseases inpatients from clinical and surgical wards and emergency section of university hospital. We characterized demographic data, presence of comorbidities, disease location and behavior, surgical past-history, extra intestinal manifestations using standardized definitions. Laboratory results were abstracted from medical records and anthropometric measures were performed during our visit. Results - The vast majority of the inflammatory bowel diseases patients had Crohn's disease (72.1\%), with ileocolic involvement (60\%), with a penetrating disease behavior (77.4\%) while ulcerative colitis group presented mostly pancolitis (50\%). Articular pain was the most common (44.2\%) extra intestinal manifestation of inflammatory bowel diseases patients and $97.7 \%$ of them had at least one type of complication related to disease. Although, the previous use of specific medical therapies to inflammatory bowel diseases before the hospitalization (more frequently corticosteroids) was done (79\%), the majority of the patients were hospitalized because of inflammatory bowel diseases activity. Disease activity was present in $80.7 \%$ of Crohn's disease and $50 \%$ ulcerative colitis patients. Inflammatory bowel diseases mortality rate was $5.5 \%$ (2/36). Comorbidities presence occurred only in $30.2 \%$ of inflammatory bowel diseases patients. The predominant surgery performed was intestinal resection. The interval between the symptoms appearance and the definitive diagnosis was less than 1 year in more than $70 \%$ of inflammatory bowel diseases patients. Laboratory findings detected were a decreased serum albumin (85.7\%) and anemia (69.8\%). The majority of the patients had at least one anthropometric alteration. The social stratification of the inflammatory bowel diseases group was similar to the Brazilian population. Conclusion - The inflammatory bowel diseases inpatients from the university hospital wards had more severe evolution of these illnesses with an active and extensive disease with complications and frequent extra intestinal manifestations, despite the prolonged use of corticosteroids. The higher prevalence of Crohn's disease inpatients than ulcerative colitis could reflect a higher aggressive behavior of this disease. The reduced serum albumin, anemia and anthropometric alterations are common inflammatory bowel diseases inpatients and could be related to a major severity of inflammatory bowel diseases evolution.

HEADINGS - Crohn disease. Proctocolitis. Hospitalization. 


\section{REFERÊNCIAS}

1. Baron S, Turck D, Leplat C, Merle V, Gower Rousseau C, Marti R, Yzet T, Lerebours E, Dupas JL, Debeugny S, Salomez JL, Cortot A, Colombel JF. Environmental risk factors in pediatric inflammatory bowel diseases: a population based control study. Gut. 2005;54:357-63.

2. Bashir S, Lipman TO. Nutrition in gastroenterology and hepatology. Prim Care 2001;28:629-45.

3. Brauer B, Korzenik RJ. Clinical features, course, and laboratory findings in Crohn's disease. In: Lichtenstein GR, editor. The clinician guide to inflammatory bowel disease. Thorofare: Slack; 2003. p.41-57.

4. Cabral VL, Carvalho L, Miszputen SJ. Importância da albumina sérica na avaliação nutricional e de atividade inflamatória em pacientes com doença de Crohn. Arq Gastroenterol. 2001;38:104-8.

5. Carter MJ, Lobo AJ, Travis SP, IBD Section British Society of Gastroenterology Guidelines for the management of inflammatory bowel disease in adults. Gut. 2004;53 (suppl 5):v1-v16.

6. Chan AT, Fleming CR, O'Fallon WM, Huizenga KA. Estimated versus measured energy requeriments in patients with Crohn's disease. Gastroenterology. 1996;91:75-8.

7. Elia PP, Tolentino YF, Luciano AL, Vargens RW, Duarte AC, Zaltman C, Fogaça HS Avalição nutricional em pacientes com doença inflamatória intestinal internados no serviço de gastroenterologia do Hospital Universitário Clementino Fraga Filho. UFRJ. In: V Semana do Aparelho Digestivo. Rio de Janeiro: Anais da V Semana do Aparelho Digestivo; 2002. p.80.

8. Elia PP, Vargens RW, Luciano AR, Barros RR, Elia C, Zaltman C, Lukashok HP, Fogaça HS. Perfil clínico, laboratorial e social dos pacientes com doença inflamatória intestinal (DII) internados em um hospital universitário na cidade do Rio de Janeiro. In: Anais do XXVIII Congreso Pan-Americano de Enfermidades Digestivas. Punta del Leste, Uruguai: 2003. p.32.

9. Faria LC, Ferrari MLA, Cunha AS. Aspectos clínicos da doença de Crohn em um centro de referência para doenças intestinais. GED Gastroenterol Endosc Dig. 2004;23:151-64.

10. Feagan BG, Sy R. Epidemiology of inflammatory bowel disease. In: Lichtenstein GR, editor. The clinician guide to inflammatory bowel disease. Thorofare: SLACK; 2003. p.1-8.

11. Fisher RL. Wasting in chronic gastrointestinal diseases. J Nutr. 1999;129 (1s suppl):252s-55s.

12. Fontanive RS, De Paula TP. Medidas antropométricas. In: Duarte AC, Castellani FR, editores. Semiologia nutricional. Rio de Janeiro: Axcel; 2002. p.33-57.

13. Forcione DG, Friedman LS. Extraintestinal manifestations of inflammatory bowel disease. In: Lichtenstein GR, editor. The clinician's guide to inflammatory bowel disease. Thorofare: Slack; 2003. p.78-112.

14. Frisancho A. New norms of upper limb fat and muscle areas for assessment of nutritional status. Am J Clin Nutr. 1981;34:2540-5.

15. Gasche C, Scholmerich J, Brynskov J, D'Haens G, Hanauer SB, Irvine EJ; Jewell DP, Rachmilewitz D, Sachar DB, Sandborn WJ, Sutherland LR. A simple classification of Crohn's disease: report of the working party for the World Congresses of Gastroenterology. Inflamm Bowel. 2000;6:8-15.

16. Gasche C, Lomer MC, Cavill I, Weiss G. Iron, anemia and inflammatory bowel diseases. Gut. 2004;53:1190-7.

17. Geerling BJ, Badart-Smook A, Stockbrügger, Brummer RJ. Comprehensive nutritional status in patients with long-stading Crohn disease currently in remission. Am J Clin Nutr. 1998;67:919-26.
18. Graham TO, Kandil HM. Nutritional factors in inflammatory bowel disease. Gastroenterol Clin North Am. 2002;31:203-18.

19. Harvey RF, Bradshaw WJM. A simple index of Crohn's disease activity. Lancet. $1980 ; 1: 5-14$

20. Jeejeebhoy KN, Hoffer LJ, Jones PJ. Clinical nutrition: management of nutritional problems of patients with Crohn's disease. CMAJ. 2002;166:913-8.

21. Kabutomori O, Kanakura Y. Movement of toxic granulation neutrophils and C-reactive protein in inflammatory processes. Am J Med. 2002;112:595-6.

22. Legnani P, Kornbluth A. Clinical features, course, and laboratory findings in ulcerative colitis. In: Lichtenstein GR, editor. The clinician's guide to inflammatory bowel disease. Thorofare: Slack; 2003. p.27-39.

23. Lichtiger S, Present DH, Kornbluth A, Gelernt I, Bauer J, Galler G, Michelassi F, Hanauer S. Cyclosporine in severe ulcerative colitis refractory to steroid therapy. N Engl J Med. 1994;330:1841-5.

24. Loftus EV Jr, Silverstein MD, Sandborn WJ, Tremaine WJ, Harmsen WS, Zinsmeiter AR. Ulcerative colitis in Olmsted County, Minnesota, 1940-1993: incidence, prevalence and survival. Gut. 2000;46:336-43.

25. Oviedo J, Farraye FA. Disease modifiers in inflammatory bowel disease. In: Lichtenstein, G.R, editor. The clinician guide to inflammatory bowel disease. Thorofare: Slack 2003. p.291-313.

26. Pinto MF. Doença inflamatória intestinal: a casuística do Hospital Universitário UFRJ [dissertação]. Rio de Janeiro: Universidade Federal do Rio de Janeiro; 1998.

27. Regueiro M. The role of endoscopy in the evalution of fistulizing Crohn's disease. Gastrointest Endosc Clin N Am. 2002;12:621-33.

28. Russel MGVM, Stockbrugger RW. Epidemiology of inflammatory bowel disease: an update. Scand J Gastroenterol. 1996;31:417-27.

29. Sampaio ARD, Mannarino IC. Medidas bioquímicas de avaliação do estado nutricional In: Duarte AC, Castellani FR, editores. Semiologia nutricional. Rio de Janeiro: Axcel; 2002.p.79-92.

30. Santos RR, Santana GO, Brito MA, Mello AC, Guedes JC, Lyra LG. Aspectos nutricionais de pacientes adultos com doença inflamatória intestinal atendidos em uma unidade de saúde de Salvador. GED Gastroenterol Endosc Dig. 2003;22:169-74.

31. Srinivasan R, Su CG, Lichtenstein GR. Medical therapy for Crohn's disease. In Lichtenstein GR, editor. The clinician guide to inflammatory bowel disease. Thorofare: Slack; 2003. p.221-54.

32. Su C, Lichtenstein GR. Recent developments in inflammatory bowel disease. Med Clin North Am. 2002;86:1497-523.

33. Su CG, Judge TA, Lichtenstein GR. Extraintestinal manifestations of inflammatory bowel disease. Gastroenterol Clin North Am. 2002;31:307-27.

34. Vatay A, Bene L, Kovacs A, Prohaszka Z, Szalai C, Romics L, Fekete B, Karadi I, Fust G. Relationship between the tumor necrosis factor alpha polymorphism and the serum C-reactive protein levels in inflammatory bowel disease. Immunogenetics. 2003;55:247-52.

35. Waitzberg DL, Caiaffa WT, Correia ITD. Hospital malnutrition: the Brazilian national survey (IBRANUTRI): study of 4000 patients. Nutrition. 2001;17:573-80.

36. Wilson A, Reyes E, Ofman J. Prevalence and outcomes of anemia in inflammatory bowel disease: a systematic review of the literature. Am J Med. 2004;116 (suppl 7A):44s-49s.

Recebido em 27/7/2005.

Reapresentado em 26/1/2007.

Aprovado em 3/4/2007. 